\title{
Segmentation and Averaging of sEMG Muscle Activations Prior to Synergy Extraction
}

\author{
Álvaro Costa-García ${ }^{\circledR}$, Eduardo Iáñez ${ }^{\circledR}$, Moeka Sonoo, Shotaro Okajima ${ }^{\circledR}$, Hiroshi Yamasaki, \\ Sayako Ueda, and Shingo Shimoda
}

\begin{abstract}
Averaging electromyographic activity prior to muscle synergy computation is a common method employed to compensate for the inter-repetition variability usually associated with this kind of physiological recording. Capturing muscle synergies requires the preservation of accurate temporal and spatial information for muscle activity. The natural variation in electromyography data across consecutive repetitions of the same task raises several related challenges that make averaging a non-trivial process. Duration and triggering times of muscle activity generally vary across different repetitions of the same task. Therefore, it is necessary to define a robust methodology to segment and average muscle activity that deals with these issues. Emerging from this need, the present work proposes a standard protocol for segmenting and averaging muscle activations from periodic motions in a way that accurately preserves the temporal and spatial information contained in the original data and enables the isolation of a single averaged motion period. This protocol has been validated with muscle activity data recorded from 15 participants performing elbow flexion/extension motions, a series of actions driven by well-established muscle synergies. Using the averaged data, muscle synergies were computed, permitting their behavior to be compared with previous results related to the evaluated task. The comparison between the method proposed and a widely used methodology based on motion flags, shown the benefits of our system maintaining the consistency of muscle activation timings and synergies.
\end{abstract}

Index Terms-Motion control, human-centered robotics, biologically-inspired robots, rehabilitation robotics.

\section{INTRODUCTION}

$\mathbf{H}$ UMAN motion is based on the complex coordination of muscles, suggesting huge computational demands from the main controller of movement, the brain [1], [2]. Yet, this is in conflict with the ease with which humans can perform everyday tasks from a proprioceptive perspective. Muscle synergies

Manuscript received September 1, 2019; accepted February 4, 2020. Date of publication February 21, 2020; date of current version March 4, 2020. This letter was recommended for publication by Associate Editor A. Ajoudani and Editor P. Rocco upon evaluation of the reviewers' comments. This work was supported by TOYOTA MOTOR CORPORATION and Japanese National Kakenhi Grants. (Corresponding author: Álvaro Costa-García.)

Álvaro Costa-García, Moeka Sonoo, Shotaro Okajima, Hiroshi Yamasaki, Sayako Ueda, and Shingo Shimoda are with the Intelligent Behaviour Control Unit (RIKEN), CBS-TOYOTA Collaboration Center in the Nagoya Science Park Research and Development Center, Nagoya, 463-0003, Japan (e-mail: acosta@brain.riken.jp; moeka.sonoo@riken.jp; shotaro.okajima@riken.jp; hym@brain.riken.jp; ueda385@brain.riken.jp; shingo.shimoda@ riken.jp).

Eduardo Iáñez is with the Brain Machine Interface Systems Lab, Miguel Hernández University (UMH), Parque Cientifico UMH, Edificio Innova, Avinguda de la Universitat s/n, 03202 Alicante, Spain (e-mail: eianez@umh.es).

Digital Object Identifier 10.1109/LRA.2020.2975729 were proposed as a solution to these paradoxical observations. According to this approach, the neural system relies on several decentralized modules (i.e., muscle synergies) that assume different tasks to partially reduce the complexity of motion controlled by the brain [3]-[6]. Even if the neural origin of the organization of muscles in synergies is still debated [7], [8], this approach is a widely used model of motor control that elegantly explains how the brain deals with the complexity of coordinating muscles during the performance of everyday tasks [9]-[12]. The use of this approach allows the extraction of the basic patterns of movement shared by many subjects from individual differences that they present when evaluating independent muscles. Traditionally, muscle synergies are computed from superficial electromyographic (sEMG) signals, which represent muscle activity, through the non-negative matrix factorization (NMF) algorithm [13]. This computational approach iteratively searches for common patterns with certain non-correlational constraints on sEMG signals. After providing a level of dimensionality reduction, the algorithm is able to express the muscle activation as a linear combination of a reduced number of patterns [14], [15].

However, there are two important factors that affect the computation of muscle synergies. The first is related to the variable nature of electrophysiological data. Over consecutive measurements, sEMG signals tend to present moderate variability. Therefore, muscle synergies extracted from independent motion periods present instabilities that complicate their evaluation. To address this issue, multiple repetitions of the movement are often averaged prior to synergy computation [16]-[18]. Thus, the study of periodic motion allows for several repetitions of a motion to be recorded, while also highlighting the effects of synergetic behavior, as such motions are based on neural processes related to automatized motions [17]. Many studies that compute muscle synergies from averaged sEMG data rely on the use of additional systems like motion tracking, inertial units, or pressure sensors in order to segment sEMG repetitions according to kinematic flags [19]-[21]. However, during periodic motions, the time between the activation of the first synergy and the deactivation of the last synergy of a motion period do not necessarily fit the kinematics of the motion. For example, after an up-down motion period is completed during elbow flexion/extension, there are still antagonist muscles fully activated to compensate for the gravitational pull that will reduce their activity during the next motion period. Accordingly, the use of kinematic information or other motion 


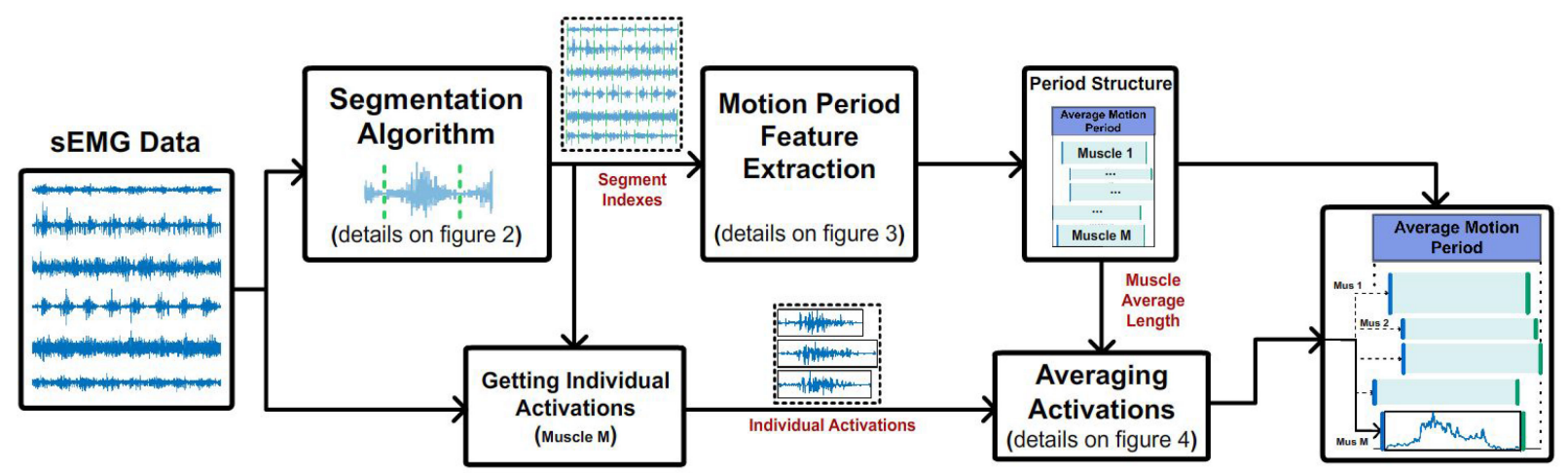

Fig. 1. PSA methodology block diagram. Block diagram representing the steps for segmenting and averaging of sEMG data. First, sEMG signals are processed with a segmentation algorithm that determines the starting and ending indexes of each individual muscle activation. On the one hand, the segment indexes from a large set of muscles are used to obtain the structure of the average motion period by extracting timing features of the motion. On the other hand, segment indexes are used to obtain individual muscle activations from the raw sEMG signal. Finally, individual muscle activations are averaged based on the average muscle lengths computed during the definition of the period structure.

events to segment sEMG data can cause inappropriate synergy segmentation.

The second factor is the conflict among the first factor (i.e., evaluation of average data), the theoretical nature of muscle synergies, and the mathematical approach used for their computation (i.e., the NMF algorithm). Synergies represent groups of muscles activated together with a specific purpose. Each synergy executes a different subtask (e.g., joint stabilization, gravity compensation, main motion pull, etc). To obtain these patterns, the time in which each muscle is triggered within the motion is a very important factor. Muscles belonging to the same synergy will activate at the same time. Therefore, changes in the activation timing of a muscle might completely modify the amount and shape of synergies required to describe the motion [22], [23]. For this reason, the variation in sEMG data between motion periods is a critical and non-trivial factor affecting the computation of muscle synergies from the averaged data.

Given the relevance of data segmentation and averaging in the process of computing muscle synergies, it is expected that some kind of standard protocol has been developed for this key task. Unfortunately, different studies use different segmentation and averaging methods, each of which are often explained poorly and do not mention how they resolve the issues raised in the present study. Even there are data alignment methodologies like manifolds [24], it is rarely apply on sEMG data. The use of a standard protocol to tackle these issues will not only improve the accuracy of synergy computation but will also increase the replicability of results based on this approach.

The present study describes both a methodology to segment sEMG data from periodic motion data (without the need for additional motion flags) and a protocol to average segmented data that have been optimized for muscle synergy extraction. As such, this is a step-by-step guide describing how to segment and average sEMG data. For pedagogical reasons, the process is divided into three stages: (a) an algorithm to segment individual trials; (b) the definition of the averaged trial size, muscle triggering timing, and duration; and (c) resampling and averaging of data. Fig. 1 shows a block diagram with the general steps followed by the proposed approach. Each block is described in detail in the Materials and Methods section.

Furthermore, the methodology is tested and validated using sEMG data recorded from 15 healthy participants during elbow flexion/extension motions.

The rest of this letter is structured as follows. The material and methods section includes information about participants, experimental protocol, sEMG recordings and methodology to track arm position during motion. After that, the methodology proposed in this letter is detailed. In addition, for comparison purposes, a widely used methodology based on motion flags is introduced. Results section shows the main outputs of applying this methodologies. On the discussion section results are evaluated in detail to extract the important points which are finally summarized in the conclusion.

\section{Materials AND MethodS}

\section{A. Participants}

From 15 participants ( 6 men and 9 women) aged 20 to 40 (mean, $26.93 \pm 6.54$ ) years, sEMG data were recorded. All participants were right-handed with no history of motor injury or dysfunction. Participants were previously informed about the experimental protocol and signed an informed consent agreement in accord with the Declaration of Helsinki. In addition, all participants were monetarily rewarded for participating in the study.

\section{B. Experimental Protocol}

Participants were asked to perform an elbow flexion/extension task at a comfortable speed. A single experimental trial included $5 \mathrm{~s}$ of relaxation followed by $20 \mathrm{~s}$ of motion. Each experimental session was composed of 4 trials. Depending on the self-selected speed of the participant, the number of motion periods in a single trial varied between 8 and 14. Overall, each participant performed between 32 and 48 elbow flexion/extension motions. Subject arm position was recorded by tracking a green ring located on their index finger with a sampling frequency of $30 \mathrm{~Hz}$. 


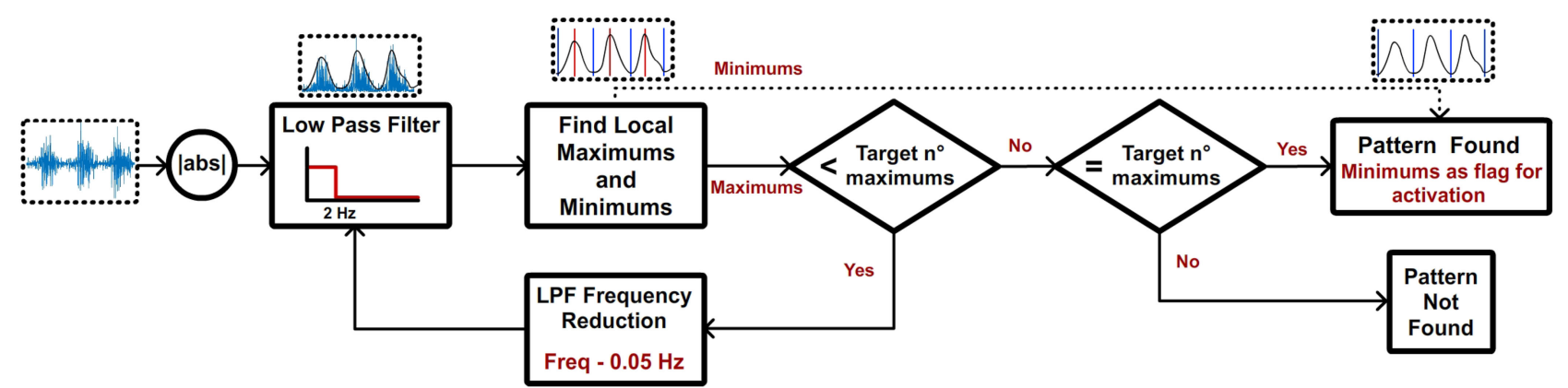

Fig. 2. Segmentation algorithm. Block diagram representing the workflow of the proposed algorithm for segmenting muscle activations using sEMG data recorded during periodic motion. The algorithm is applied individually to sEMG signals from each muscle. First, data are rectified and low-pass filtered (2 Hz). Then, local maxima of filtered data are compared with the expected number of muscle activations (provided manually). If there are more maxima detected than expected, the cutting frequency of the low-pass filter is reduced in order to find a pattern that better describes the motion. This process is repeated until the algorithm finds a pattern that fits the motion (establishing the minima of that pattern as the segmentation points). If such a pattern is not found, it is assumed that the muscle under evaluation does not actively affect the motion.

Visual tracking algorithms were implemented by the modular toolbox for interactive systems (Bonsai, NeuroGEARS) [25]. Fig. 5 shows the experimental environment. Arm position is tracked with the side camera and a graphical interface is presented in front of the user to maintain speed stability between consecutive motions.

\section{C. sEMG Recording}

All sEMG signals were acquired using 6 wireless sensors (BTS FREEEMG; BTS Bioengineering Corp., Milan, Italy) placed on the right arm of each participant on the following muscles: wrist flexor (WF), pronator teres (PT), wrist extensor (WE), biceps (B), triceps (T), and anterior deltoid (AD). Electrodes were placed according to the guidelines of the Surface Electromyography for the Non-Invasive Assessment of Muscles (SENIAM) project [26]. Data were recorded from bipolar sensors with a sampling frequency of $1000 \mathrm{~Hz}$. A $60-\mathrm{Hz}$ notch filter was used to remove the signal emanating from the power source.

\section{Proposed Segmentation and Averaging Methodology (PSA)}

1) Segmentation Algorithm: Fig. 2 shows the workflow of the algorithm used to segment independent muscle activations during the performance of the task. The algorithm uses as input the sEMG data associated with the activation of a muscle and the number of times that the task was repeated.

On the first iteration, before rectification, a $2-\mathrm{Hz}$ low-pass filter is applied to the data and the local maxima and minima of the filtered signal are computed. A local minimum is defined as a sample neighbored by two larger samples and a local maximum is defined as a sample that is larger than its two neighboring samples. Next, the number of local maxima detected within the motion range is compared with the expected number of repetitions. At this point there are three possible outputs:

a) If the values are equal, the algorithm found a pattern within the signals that repeats with the same frequency as the expected muscle activations. The local minima computed on that iteration are then chosen as segmentation points for the evaluated data. b) If the number of local maxima exceeds the number of expected activations, the algorithm assumes that the frequency of the low-pass filter is too high to fit the frequency of the motion. Thus, the process starts again after reducing the frequency of the low-pass filter by $0.05 \mathrm{~Hz}$.

c) If the number of expected muscle activations exceeds the number of local maxima, the algorithm is unable to find a pattern that fits the expected number of repetitions. In that scenario, it is assumed that the electrode under analysis does not contribute to the motion with a periodic activation.

For each electrode, this provides a vector of local minima fitting the activation timing of the muscle during the task. In the current methodology, $2 \mathrm{~Hz}$ was chosen as the initial value of the low-pass filter because periodic tasks performed by humans do usually not exceed this motion frequency [27]. However, this value might be modified depending on the task under evaluation. In addition, the frequency reduction of each iteration (in this case $0.05 \mathrm{~Hz})$ can be also modified according the features of the data.

2) Definition of a Motion Period: Defining an average motion period requires the definition of its length and the timings in which muscles are contracting within one period. However, human periodic motions are not ideally periodic. In each repetition there are small deviations in the activation timings. Accordingly, consecutive activations of the same muscle during periodic motion vary in the number of samples (i.e., the activations in each row of Fig. 3). In addition, when evaluating the whole set of muscles within one period (i.e., each column of Fig. 3), the starting and ending points of the activation of each muscle also differ. Given these conditions, the average motion period was computed through the following steps:

1) Each activation was defined between the beginning of the first triggered muscle (red dotted line in Fig. 3) and the end of the last muscle deactivation (pink dotted line in Fig. 3)

2) After repeating this process for each activation (i.e., each column of Fig. 3), the median starting and ending values of each muscle are used as starting and ending points of the same muscle for the average motion period.

3) The length of the averaged motion and the time-shift between muscles is determined by the starting and ending points defined in the previous step. 

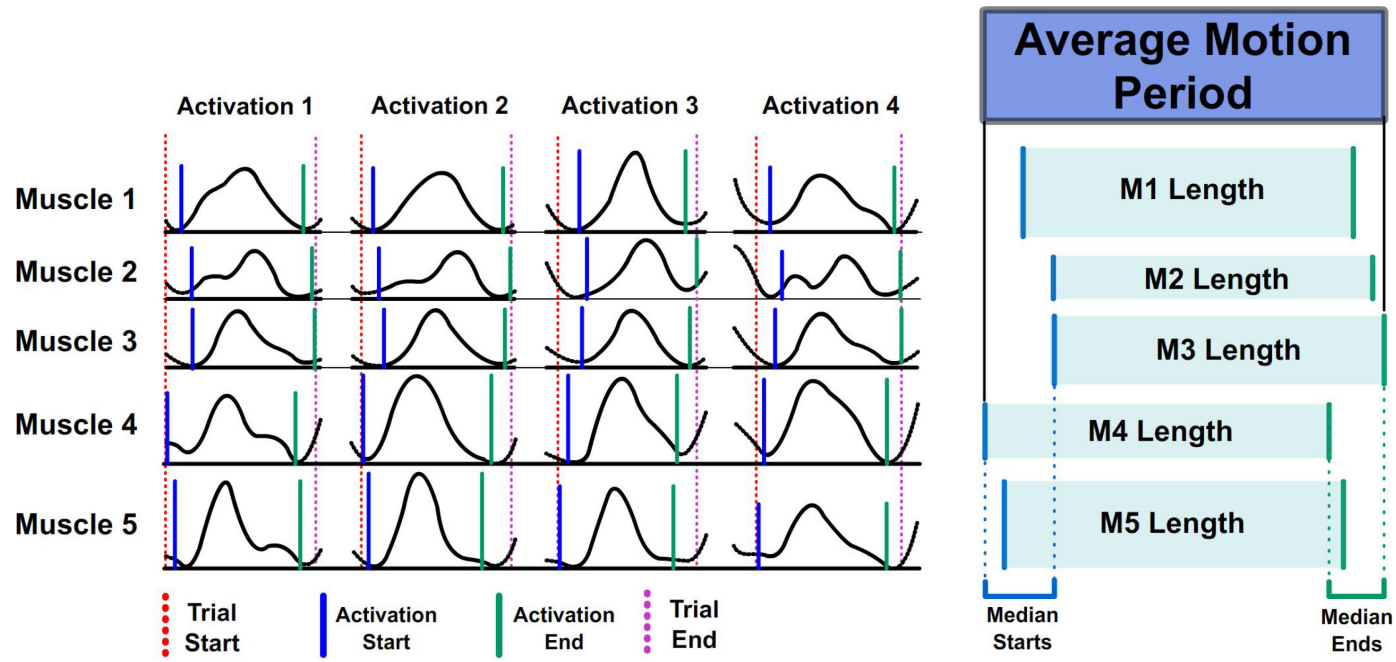

Fig. 3. Average motion period. Representation of the average motion period using starting and ending points of consecutive muscle activations. For each single activation, the duration of the motion is defined as the time between the first muscle activation and the last muscle deactivation. The starting point of a muscle in the average motion period is computed as the median value of all the starting points of that muscle during each independent activation. The same process is followed to compute the ending points of each muscle. Based on the starting and ending points of all muscles, the length of each muscle activation and the total length of the average period are defined.

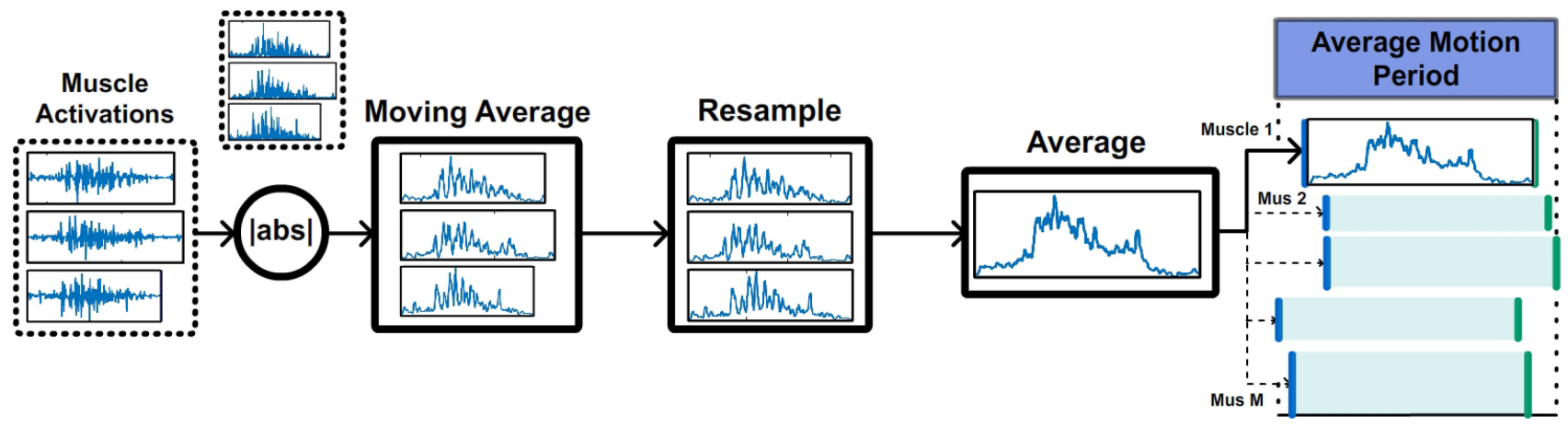

Fig. 4. Resampling and averaging. Representation of the process for averaging the data from each muscle. Individual muscle activations are rectified and a moving average filter is applied to emphasize the low frequencies that modulate muscle contractions. Each activation is resampled according to average duration of the corresponding muscle (defined on Fig. 3). The mean value of the resampled activation is included into the averaged motion period. This process is repeated for each muscle.

As in step (1), the average trial length is defined as the time between the first muscle activation and the last muscle deactivation.

3) Trial Averaging: To obtain the average trial, the empirical sEMG data must be fit to the average motion period dimensions defined previously. Fig. 4 illustrates this process. Thus, independent activations associated with the same muscle are rectified, and a moving average filter (20 samples, $0.02 \mathrm{~s}$ ) is applied to the signals to highlight the low frequencies modulated by muscle activity. Filtered trials are resampled to fit the dimensions associated with the muscle being analyzed [28]. The resampling rate is computed as the ratio between the length of each single activation and the muscle length associated with the average motion period. Finally, resampled trials are averaged and included in the corresponding muscle slot according to the position defined by the average motion period. This process is repeated for each muscle contributing to the motion.

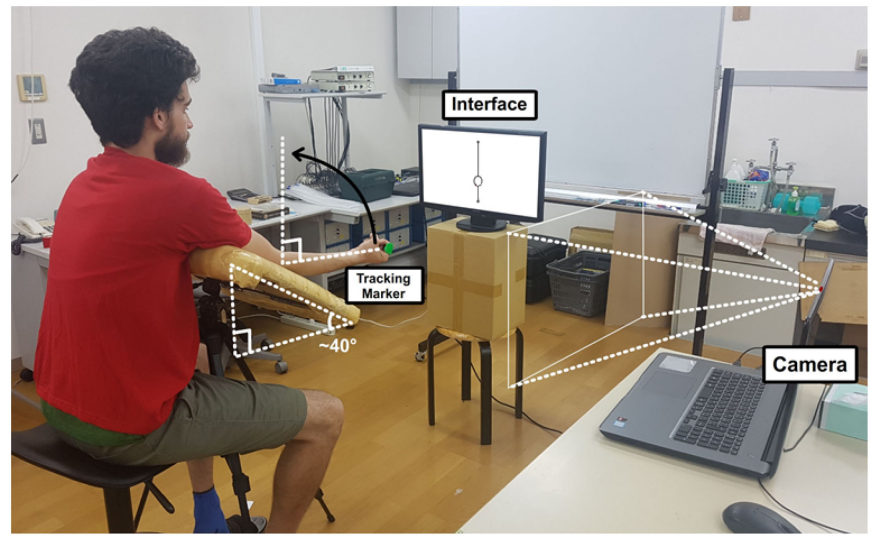

Fig. 5. Experimental environment. Participant's arm is supported with an inclination of 40 degrees. A lateral camera is tracking the arm position using a green ring located in the index finger as reference. A visual interface shows a ball moving up and down at a constant speed (defined in accordance with the comfortable speed chosen by the subject). 


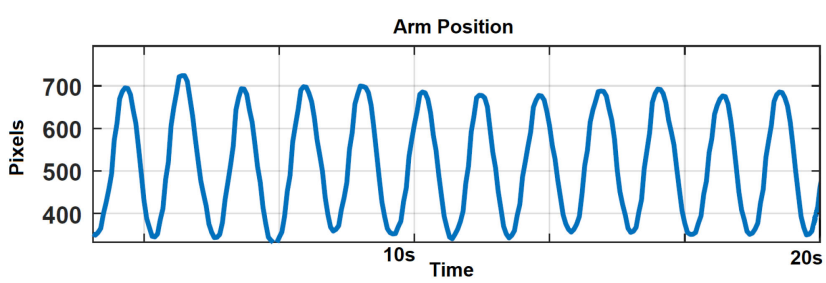

Fig. 6. Arm Position. Representation of the arm position in pixels as the addition of $\mathrm{x}$ and $\mathrm{y}$ position of the arm during elbow flexion/extension. The position of maximum elbow extension was fixed for lower $\mathrm{x}$ and $\mathrm{y}$ values and maximum elbow flexion was fixed for higher $\mathrm{x}$ and $\mathrm{y}$ values. Minima in the graph represent extended arm and maxima represent flexed arm.

\section{E. Motion Flags Segmentation and Averaging Method (FSA)}

As mention before, a common strategy to segmentate sEMG data is based on the use of flags that define key points of the motion [16]-[18]. In this case, the arm position will be used as flag. Fig. 6 shows an example of the data provided by the tracking algorithm showing clearly the points of maximum and minimum position of the arm. Maximum and minimum values will be used to cut the individual repetitions on the sEMG data. Each repetition will be resampled to the median repetition size and averaged. This process is repeated for each subject independently.

\section{F. Synergy Computation}

Averaged signals should be easily decomposed into two synergies representing the up and down phase of elbow flexion/extension motion. The muscle synergies were defined as

$$
\begin{array}{cl} 
& M=W \cdot C, \\
M \epsilon R^{m \times t}, & W \epsilon R^{m \times n}, \quad C \in R^{n \times t},
\end{array}
$$

where $M$ is an $m \times t$ matrix of sEMG data (with $m$ being the number of channels and $t$ the number of samples), $W$ is an $m \times n$ matrix containing the muscle synergies used to reduce the $m$ channels to an $n$-dimensional space, and $C$ is an $n \times$ $t$ matrix containing the $n$ simple patterns used to control the whole set of $m$ channels evaluated. Matrices $C$ and $W$ can be calculated from $M$ through using the NMF algorithm by fixing the $n$-dimensionality desired (in this case $n=2$ synergies).

\section{G. Average Synergy Similarity Coefficient}

Synergies for elbow flexion/extension have been widely analyzed in literature and their temporal and spatial patterns present many similarities among different subjects. Therefore, evaluating the deviation between each subject synergies and the average synergies of all subject provides an efficient parameter to decide with set of synergies represent better the motion under evaluation. The Average Synergy Symilarity (ASS) coefficient on equation 3 was used to perform this evaluation.

$$
A S S_{u}=\frac{\sum_{s y n=1}^{n \_s y n} \frac{\operatorname{corr}\left(H_{s y n}, \overline{H_{s y n}}\right)+\operatorname{corr}\left(W_{s y n}, \overline{W_{s y n}}\right)}{2}}{n \_s y n},
$$

$A S S_{u}$ represents the ASS coefficient for the subject $u$ where $\operatorname{corr}\left(H_{\text {syn }}, \overline{H_{\text {syn }}}\right)$ is the correlation coefficient between the

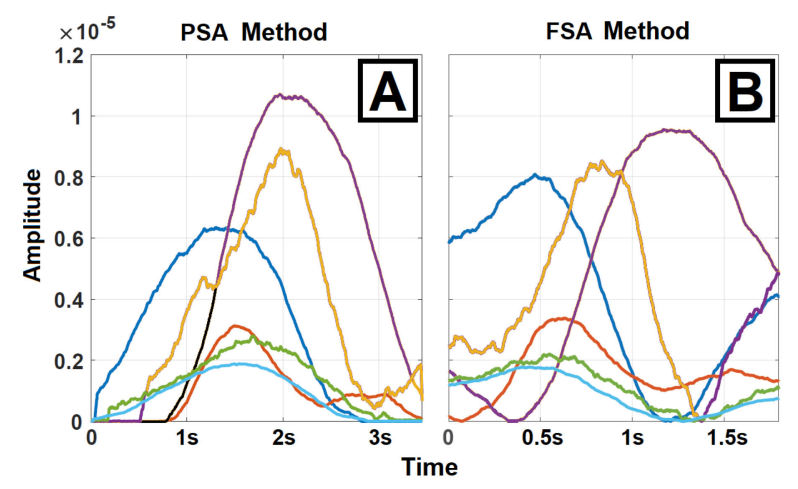

Fig. 7. Averaged sEMG signals. Example of the average activations of each muscle for the 1 participant: blue, wrist flexor (WF); orange, pronator teres (PT); yellow, wrist extensor (WE); purple, biceps (B); green, triceps (T); cyan, anterior deltoid (AD). Graph A shows the average computed by PSA method. Graph B shows the average data obtained by FSA method.

temporal pattern of the synergy number syn $\left(H_{\text {syn }}\right)$ and the temporal pattern of same synergy averaged from all subjects $\left(\overline{H_{s y n}}\right)$. The same process is followed to compute the similarity among the spatial patterns $\left(\operatorname{corr}\left(W_{\text {syn }}, \overline{W_{\text {syn }}}\right)\right)$. Finally, $n \_s y n$ represents the number of synergies extracted, 2 in this case.

\section{RESULTS}

\section{A. Averaged sEMG Signals}

Fig. 7 shows the average values computed for the recorded muscles using the PSA (Fig. 7A) and FSA (Fig. 7B). Graphs represent, as an example, the data from the same subject after applying both methodologies. As expected, the biceps was the main muscle associated with the flexion motion, and extension was strongly associated with the wrist extensor. The functions of the remaining muscles varied across participants, highlighting the wide range of strategies available for motor control. In Fig. $7 \mathrm{~A}$ all muscles were resampled according to their median lengths and located within the motion period according to their median starting and ending points across all trials. The beginning and ending points of the signal define clear muscle activation and deactivations, which is helpful for identifying synergies. In Fig. 7 B repetitions were segmented according maximum and minimum positions of the arm during elbow flexion. Repetitions were also resampled according to the median repetition length but timings of activation and deactivation of individual muscles was not taken into account during averaging. As a result apart from the clear decoupling among muscles, the activation and deactivation timing are less clear as muscle activation do not correspond with minimum and maximum values of arm position.

\section{B. Synergies}

Fig. 8 shows the synergies (time coefficients and weights) computed for the set of averaged sEMG signals shown on Fig. 7. Fig. 8 A shows the synergies computed from the signal averaged with PSA while Fig. 8 B shows the synergies obtained from FSA average data. In addition, the contribution of each muscle and synergy to the motion is represented by the bar graph of weights shown beside each line graph. The results from Fig. 8 A are 


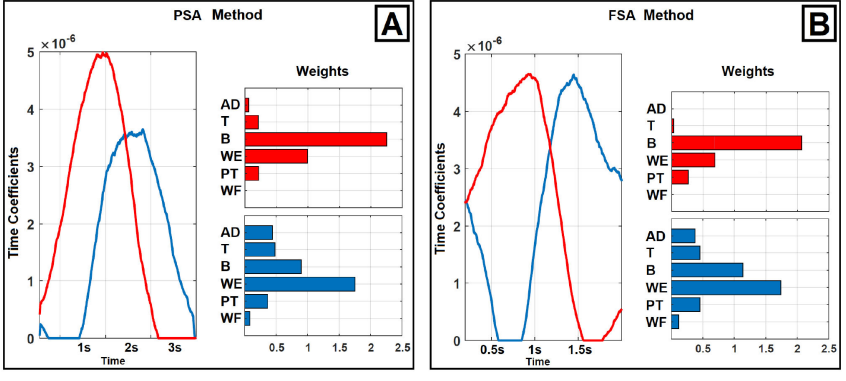

Fig. 8. Muscle synergies. Muscle synergies extracted from the averaged sEMG data shown in Fig. 7. Coefficients represent the time distribution of power exerted during the motion period. Weights represent the spatial distribution of the power (i.e., how the muscle activations were distributed among the recorded muscles. Graph A shows the synergies extracted from the averaged sEMG computed by PSA. Graph B shows the synergies extracted from average sEMG obtained by FSA.)

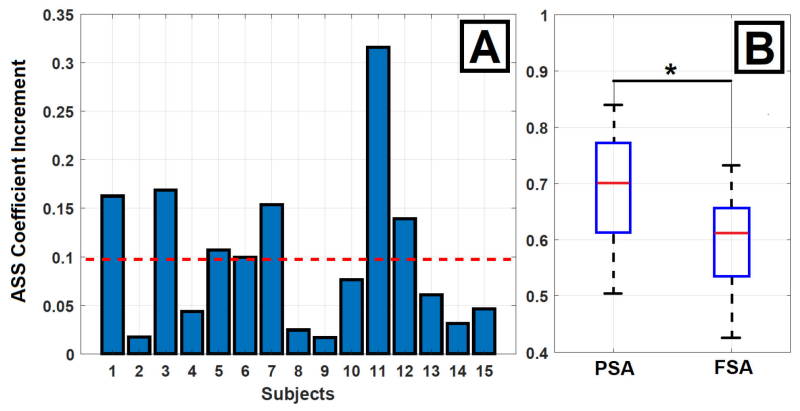

Fig. 9. Average Synergy Similarity Comparison. Graph A shows, for each subject, the difference between ASS index computed for the synergies extracted using the PSA and FSA. Red slashed line represent average value of the distribution. Graph B shows the distribution of coefficients for each method and its significance using a Wilcoxon sum-rank test with a confidence interval of $95 \%$.

in accordance with the published literature; namely, the flexion synergy was associated with the biceps and synergetic muscles carried most of the motion effort, while a second synergy was activated during the extension to create a smooth motion downward by compensating for gravitational pull [29]-[31]. In the case of Fig. 8 B, even spatial patterns do not strongly differ from those obtained on $8 \mathrm{~A}$, time patterns are decoupled making more complicated to identify the activation timings of each synergy.

\section{ASS Coefficient}

The ASS coefficient was computed on the synergies extracted from PSA and FSA methodologies. Fig. 9 A shows the difference between the ASS coefficient computed FSA and PSA. Positive values on this graph show an improve of PSA synergies compared to FSA synergies. Fig. 9 B show each set of ASS represented as a boxplot. A Wilcoxon sum-rank test with a confidence interval of $95 \%$ was perform to confirm the statistical significance between both groups.

\section{DISCUSSION}

The segmentation algorithm proposed in section II-D1 does not require the use of motion flags from motion tracking, inertial sensors, or similar systems. The algorithm relies on the low frequency patterns modulated by muscle fibers during motion and just requires a priori knowledge of the number of repetitions performed during the experiment. Through an iterative filtering process, it was possible to find this pattern and use its minimum values to segment the data. In addition, the algorithm works independently on each contributing muscle, thus maintaining their temporally shifting relationships with the motions. Independent muscle segmentation is completely detached from kinematics, enabling a clear definition of a motion period and, therefore, the proper identification of the range of data representing a single activation of a set of synergies.

With sEMG data segmented this way, each single muscle activation can be evaluated in two ways. The first one is as a part of the set of muscle activations that generate one motion period (i.e., the columns in Fig. 3). From this evaluation, instant period length can be computed together with the duration and triggering of the muscle composing it. The second evaluation can be made among consecutive activations of the same muscle (rows of Fig. 3). From this analysis, the median starting and ending points of each muscle can be computed, thereby defining the basic structure of an average motion period. This way of defining the average motion period robustly conserves the temporal information capturing muscle triggering time and duration. As noted above, this is a crucial factor for the computation of synergies [22], which are defined as groups of muscle triggered together for the same physiological purpose.

Inferring the individual activations and their average lengths greatly simplifies resampling and averaging of the sEMG data for each muscle as represented in Fig. 4. Moreover, the average result can be easily shifted to the appropriate timing guided by the average motion period.

The comparison of PSA with FSA (in which sEMG data is segmentated based on arm position and inter-repetition muscle activation time shifting is not contemplated) shows clear time decoupling in the final averaged signals. Moreover, synergies extracted with PSA present less deviation from their average values.

The proposed method enables the isolation of a single set of muscle activations within a motion period, while keeping the temporal information for each muscle and without including extra information from previous and subsequent activations (Fig. 7A). The computation of muscle synergies from this averaged data provides clear activation patterns associated with the sets of muscles contributing to each part of the motion.

\section{CONCLUSION}

This works presented a step-by-step protocol for the segmentation and averaging of sEMG signals prior to muscle synergy computation. The presented methodology was developed to handle two challenges emerging from averaging periodic motions: (a) the isolation of independent muscle activation using only sEMG data and (b) choosing the dimensions of the average trial to comply with the temporal and spatial information associated with muscle activations required to extract accurate muscle synergies. The protocol was validated with sEMG data from 15 healthy participants who performed periodic elbow 
flexion/extension motions. Average trials exhibited smooth activations, and the inferred muscle synergies were in accordance with the current literature on elbow flexion/extension tasks. Moreover, independent muscle activation durations and triggering times could be obtained from the segmentation methodology described in this work. These values can be used to evaluate the standard variability in physiological data recorded from healthy individuals. Using these data as a ground truth should facilitate the study of different motor disorders that affect these parameters. The methodology introduced in this letter will be used in future research involving the comparison of muscle synergies for the same task under different experimental conditions. Establishing a standard method for averaging trials enables more accurate task comparisons and increases the replicability of experimental results.

\section{REFERENCES}

[1] J. C. Ives, Motor Behavior: Connecting Mind and Body for Optimal Performance. Lippincott Williams \& Wilkins, 2013.

[2] B. Abernethy and W. Sparrow, "The rise and fall of dominant paradigms in motor behaviour research," Advances Psychol., vol. 84, pp. 3-45, 1992.

[3] M. Jeannerod, The Neural and Behavioural Organization of Goal-Directed Movements. Oxford, U.K.: Clarendon, 1988.

[4] E. Pierrot-Deseilligny and D. Burke, The Circuitry of the Human Spinal Cord: Its Role in Motor Control and Movement Disorders. Cambridge, MA, USA: Cambridge Univ. Press, 2005.

[5] F. Shibata Alnajjar, T. Wojtara, H. Kimura, S. Shimoda, "Muscle synergy space: Learning model to create an optimal muscle synergy," Frontiers Comput. Neuroscience, vol. 7, pp. 1-10, 2013.

[6] Á. Costa, M. Itkonen, F. S. Alnajjar, and S. Shimoda, "Importance of muscle selection for EMG signal analysis during upper limb rehabilitation of stroke patients," in Proc. IEEE 39th Аnnu. Int. Conf. Eng. Med. Biol. Soc., 2017, pp. 2510-2513.

[7] M. C. Tresch and A. Jarc, "The case for and against muscle synergies," Current Opinion Neurobiology, vol. 19, no. 6, pp. 601-607, 2009.

[8] Á. Costa-García, et al., "Model for prompt and effective classification of motion recovery after stroke considering muscle strength and coordination factors," J. Neuroengineering Rehabil., vol. 16, no. 1, pp. 1-11, 2019.

[9] A. d'Avella, P. Saltiel, and E. Bizzi, "Combinations of muscle synergies in the construction of a natural motor behavior," Nature Neuroscience, vol. 6, no. 3 , pp. 300-308, 2003.

[10] G. Torres-Oviedo and L. H. Ting, "Subject-specific muscle synergies in human balance control are consistent across different biomechanical contexts," J. Neurophysiology, vol. 103, no. 6, pp. 3084-3098, 2010.

[11] N. Yang et al., "Temporal features of muscle synergies in sit-to-stand motion reflect the motor impairment of post-stroke patients," IEEE Trans. Neural Syst. Rehabil. Eng., vol. 27, no. 10, pp. 2118-2127, Oct. 2019.

[12] H. Kogami et al., "Effect of physical therapy on muscle synergy structure during standing-up motion of hemiplegic patients," IEEE Robot. Automat. Lett., vol. 3, no. 3, pp. 2229-2236, Jul. 2018.

[13] M. C. Tresch, V. C. Cheung, and A. d'Avella, "Matrix factorization algorithms for the identification of muscle synergies: Evaluation on simulated and experimental data sets," J. Neurophysiology, vol. 95, no. 4, pp. 2199-2212, 2006.
[14] Y. Zhang and Y. Fang, "A NMF algorithm for blind separation of uncorrelated signals," in Proc. Int. Conf. Wavelet Anal. Pattern Recognit., 2007, vol. 3, pp. 999-1003.

[15] Á. Costa-García, M. Itkonen, H. Yamasaki, F. Shibata-Alnajjar, and S. Shimoda, "A novel approach to the segmentation of sEMG data based on the activation and deactivation of muscle synergies during movement," IEEE Robot. Autom. Lett., vol. 3, no. 3, pp. 1972-1977, Jul. 2018.

[16] R. Ranganathan and C. Krishnan, "Extracting synergies in gait: Using EMG variability to evaluate control strategies," J. Neurophysiology, vol. 108, no. 5, pp. 1537-1544, 2012.

[17] G. Courtine, C. Papaxanthis, and M. Schieppati, "Coordinated modulation of locomotor muscle synergies constructs straight-ahead and curvilinear walking in humans," Exp. Brain Res., vol. 170, no. 3, pp. 320-335, 2006.

[18] A. Hof, H. Elzinga, W. Grimmius, and J. Halbertsma, "Speed dependence of averaged EMG profiles in walking," Gait Posture, vol. 16, no. 1, pp. 78$86,2002$.

[19] J. Gonzalez-Vargas, M. Sartori, S. Dosen, D. Torricelli, J. Pons, and D. Farina, "A predictive model of muscle excitations based on muscle modularity for a large repertoire of human locomotion conditions," Frontiers Comput. Neuroscience, vol. 9, pp. 1-14, 2015.

[20] M. H. van der Linden, D. S. Marigold, F. J. M. Gabreëls, and J. Duysens, "Muscle reflexes and synergies triggered by an unexpected support surface height during walking," J. Neurophysiology, vol. 97, no. 5, pp. 3639-3650, 2007.

[21] A. J. Meyer, I. Eskinazi, J. N. Jackson, A. V. Rao, C. Patten, and B. J. Fregly, "Muscle synergies facilitate computational prediction of subjectspecific walking motions," Frontiers Bioengineering Biotechnology, vol. 4, pp. 1-26, 2016

[22] I. Delis, S. Panzeri, T. Pozzo, and B. Berret, "A unifying model of concurrent spatial and temporal modularity in muscle activity," J. Neurophysiology, vol. 111, no. 3, pp. 675-693, 2013.

[23] P. Kieliba, P. Tropea, E. Pirondini, M. Coscia, S. Micera, and F. Artoni, "How are muscle synergies affected by electromyography preprocessing?" IEEE Trans. Neural Syst. Rehabil. Eng., vol. 26, no. 4, pp. 882-893, Apr. 2018.

[24] C. Wang and S. Mahadevan, "A general framework for manifold alignment," in Proc. AAAI Fall Symp. Series, 2009, pp. 1-8.

[25] G. Lopes et al., "Bonsai: An event-based framework for processing and controlling data streams," Frontiers Neuroinformatics, vol. 9, pp. 1-14, 2015.

[26] H. J. Hermens et al., "European recommendations for surface electromyography," Roessingh Res. Develop., vol. 8, no. 2, pp. 13-54, 1999.

[27] R. B. Alderman, "Age and sex differences in learning and performance of an arm speed motor task," Res. Quart. Amer. Assoc. Health, Phys. Edu. Recreation, vol. 39, no. 3, pp. 428-431, 1968.

[28] N. Abser, D. MacIsaac, A. Chan, G. Fraser, and J. R. Green, "CleanEMG: Comparing interpolation strategies for power line interference quantification in surface EMG signals," in Proc. Int. Conf. Can. Med. Biol. Eng. Soc. Proc., vol. 35, 2012, pp. 1-4.

[29] E. Chiovetto, B. Berret, I. Delis, S. Panzeri, and T. Pozzo, "Investigating reduction of dimensionality during single-joint elbow movements: A case study on muscle synergies," Frontiers Comput. Neuroscience, vol. 7, pp. $1-$ $12,2013$.

[30] J. C. Jamison and G. E. Caldwell, "Muscle synergies and isometric torque production: Influence of supination and pronation level on elbow flexion," J. Neurophysiology, vol. 70, no. 3, pp. 947-960, 1993.

[31] S. Le Bozec, B. Maton, and J. Cnockaert, "The synergy of elbow extensor muscles during dynamic work in man," Eur. J. Appl. Physiol. Occupational Physiol., vol. 44, no. 3, pp. 255-269, 1980. 